\title{
Ketamine’s Journey from Sedation to Suicide Prevention: A Viewpoint
}

\section{Saeed Ahmed ${ }^{1^{*}}$, Mudasar Hassan², Hema Venigalla ${ }^{3}$, Hema Madhuri Mekala and Mustafa Qureshi ${ }^{5}$}

${ }^{1}$ Nassau University Hospital, New York, United States

${ }^{2} A$ \& L Physicians, New York, United States

${ }^{3}$ Texas Behavioral Health Clinic, Texas, United States

${ }^{4}$ University of Missouri, Missouri United States

${ }^{5}$ Zucker Hillside Hospital LIJ, New York, United States

\begin{abstract}
Suicide is the tenth leading cause of death in the United States. According to research in 2016, the suicide rate has increased substantially both in the United States and worldwide. Most people who commit suicide are found to suffer from psychiatric illnesses like depression, schizophrenia, anxiety, bipolar disorder, alcoholism, and posttraumatic stress disorder (PTSD). In the past several studies are conducted to examine which drugs decrease suicidal ideation in mentally ill patients. Selective serotonin reuptake inhibitors (SSRIs) like Prozac (fluoxetine) are believed to decrease suicidal intentions in patients with depressive disorders. Similarly, lithium and clozapine are effective in reducing suicidality in patients with bipolar depression and schizophrenia, respectively. Although these drugs have a demonstrated efficacy to reduce suicidality long-term, there is limited evidence that these drugs have the same effect during the acute phase of illness. Most psychotropic medications take a few weeks to work; unfortunately, some patients require sufficiently more time to stabilize their symptoms. Because time is a crucial factor when dealing with suicidal patients, we express our viewpoint regarding the use of ketamine, which may, within hours or days help patients with suicidal ideation.
\end{abstract}

\section{Suicidality and Mental Health}

Every year approximately 37,000 people in the US die from suicide [1], and one million die worldwide [2]. In the US, each year 650,000 people receive emergency treatment after attempting suicide [2]. Suicide still leads the nation as one of the principal causes of death. According to an April 2016 report from the Centers for Disease Control and Prevention, the suicide rate has increased substantially (24\%) in the 15 years from 1999 to 2014 in the United States. Analogously, global suicide rates have increased $60 \%$ in the past 45 years. Consistently, $90 \%$ of suicides are by people found to suffer from psychiatric illnesses: depression, schizophrenia, anxiety, bipolar disorder, alcoholism, PTSD.

Although the exact mechanism leading to suicidal ideation is unknown, this psychiatric dilemma influenced by a complex congruence of biological, psychological, and social factors might be the prime cause. At any rate, both psychological factors and psychiatric disorders seem to impose a maximum toll, cascading to increased suicidality. Almost all psychiatric disorders engender suicidal predispositions; however, the cognitive processes leading to the taking of one's life may vary. Irrespective of any varied thoughts, the pathogenesis for suicidal intentions is mainly linked to altered and distorted levels of various brain chemicals [3].

Suicidal ideation is a common presenting symptom of psychiatric patients and is the primary reason for hospitalization. Psychiatrists and psychologists are specifically trained to help these patients by identifying those who are at risk for suicide and treating them with psychotropic management, counseling, therapy, and other treatment modalities. Needless to say, almost all the psychiatric disorders like depression, anxiety disorder, bipolar disorder, PTSD, and schizophrenia predispose the patients to have both passive and active suicidal thoughts [4].

Clearly, it is incumbent upon the doctor to identify and then prevent suicide when dealing with psychiatric patients. Risk factors like past suicide attempts, family history, access to guns and other weapons, life stressors, adverse personal experiences (rape, sexual abuse), and terminal illness should be carefully evaluated and addressed appropriately through counseling or hospitalization, if necessary [5]. The core care team of physicians, therapists and healthcare personnel are responsible for identifying potential patients for suicide risk and encouraging them to discuss their problems with their family or doctors, essentially circumventing suicide via more appropriate solutions. At the same time, psychotropic management tailored to the specific condition together with regular cognitive behavioral therapy (CBT) can lead to decreased suicidality in this patient population. It is of vital importance that psychiatrists work steadfastly toward encouraging patients to comply with medication and CBT for optimal results. Moreover, patients should be educated on the risks associated with abrupt cessation of pharmacologic therapy as such might induce increased suicidality.

\section{Pharmacotherapy Decreasing Suicidality}

Several studies so far have substantiated that lithium has positive effects on decreasing suicidality on a long-term basis not only for bipolar disorder but also major recurrent depression and other manicdepressive illnesses [6]. According to Baldessarini et al. attempted and completed suicide rates are significantly reduced with lithium treatment (Suicides: 0.16; Attempts: 0.41) compared to those without (suicides: 0.88; Attempts: 4.02) in patients with bipolar and recurrent depressive disorders [7]. Clozapine is the first FDA-approved antipsychotic that can mitigate suicidality in patients with schizophrenia and schizoaffective disorder. Indeed, Meltzer reported that the suicide rate decreased by $88 \%$ in patients with schizophrenia after two years of clozapine treatment [8]. Quetiapine has also been shown clinically to reduce the suicide risk

*Corresponding authors: Saeed Ahmed, Department of Psychiatry, Nassau University Medical Center, East Meadow, NY 11554, USA, Tel: (516) 572-0123. E-mail: ahmedsaeedmd@gmail.com

Received: September 02, 2016; Accepted:September 15, 2016; Published: September 22, 2016

Citation: Ahmed S, Hassan M, Venigalla H, Mekala HM, Qureshi M (2016) Ketamine's Journey from Sedation to Suicide Prevention: A Viewpoint. J Psychiatry 19: 383. doi:10.4172/2378-5756.1000383

Copyright: ( 2016 Ahmed S, et al. This is an open-access article distributed under the terms of the Creative Commons Attribution License, which permits unrestricted use, distribution, and reproduction in any medium, provided the original author and source are credited. 
in bipolar and psychotic patients for whom clozapine is contraindicated [9]. Insofar as medications to treat depression, SSRIs are believed to be one of the best lines of defense. As far as the SSRI class, few studies supported that chronic use reduced the rate of completed suicide in patients with depression. Of all the SSRIs, fluoxetine is found to be more effective in reducing suicidal behavior in these patients [10]. Although the drugs mentioned have been clinically shown to decrease suicidality in long-term, the concern remains about decreased suicide-risk during the acute phase of the illness. Evidently, during this period, patients are at a significantly higher risk for attempting suicide, and this timeline makes it extremely crucial that suicidal intervention is addressed.

There is evidentiary support in the published literature that drugs like ketamine may help within hours or days in patients with suicidal ideation. Recently, ketamine has been proposed to reduce the suicide risk in these acutely depressed patients. A study by Zarate et al. showed that intravenous infusion of ketamine could decrease suicidal ideation within hours to days in a hospital setting, especially in patients with treatment-resistant depression. In this same study, the patients who received ketamine did experience adverse side-effects like confusion, high blood pressure, dizziness, euphoria, increased libido, and perceptual disturbances [11]. In another study by Kashani et al. ketamine was evaluated for its effects on 49 patients who were admitted with suicidal behavior to the emergency department. The researchers discovered that significant score reduction was obtained on both the Scale for Suicidal Ideation (SSI) and the MontgomeryAsberg Depression Rating Scale (MADRS), 40, 80 and 120 minutes after ketamine infusion [12]. Despite the above evidence, ketamine use may alter brain chemicals in a manner similar to stimulant drugs, and its administration might become addictive. Consequently, such drugs must be prescribed with caution until more evidence-based research is established. To follow is a discussion of suicidality and the use of ketamine as an agent to ameliorate this concern acutely and also outweigh its benefits vs risks in clinical application.

\section{Discussion}

As already stated, suicidal ideation and behavior are the most common yet gravest psychiatric emergencies. Accordingly, the drugs lithium, clozapine, and fluoxetine have proven effective to reduce suicidal risk. Unfortunately, there is no one psychotropic agent that addresses this concern irrespective of the diagnosis for long-term prevention. Although ketamine has been shown to take effect quickly to reduce suicidal ideation in the emergency room and hospital settings, there are many concerns regarding its administration in practice. Ketamine can cause drowsiness, disorientation, hallucinations, confusion, panic attacks, and anxiety after infusion. Furthermore, chronic ketamine usage is known to have several adverse effects: mood and personality changes, depression, flashbacks, psychosis, disrupted learning, and impaired memory processing. Beyond these side-effects, the main drawback of ketamine is its potential to be abused, which can cause toxic effects similar to stimulant drugs and lead to physiological and psychological dependence. Thus, using ketamine to treat suicidal behavior in patients with known histories of drug addiction is a debatable issue.

Additionally, the acute management of hospitalized suicide patients with IV-ketamine infusion can be daunting given all the safety monitoring measures for this drug. On an out-patient basis, the clinical application of ketamine to treat high-risk, actively suicidal patients remains controversial as ketamine has not demonstrated therapeutic value to treat psychiatric disorders. Also, there is no study to show that decreased suicidality achieved from the acute administration of ketamine can be maintained further via ketamine itself. For this reason, larger prospective studies are required to demonstrate the therapeutic efficacy of ketamine to decrease sucidality both short and long term. An important determination, as well, would be whether the benefits of ketamine outweigh the risks when using the drug in clinical settings. Ultimately, much more research is needed to better understand the pathogenesis of brain chemical alteration in suicidal patients and to come up with novel options to target these drugs for both prevention and treatment of suicidal behavior.

\section{References}

1. Murray CJ, Atkinson C, Bhalla K, Birbeck G, Burstein R, et al. (2013) The state of US health, 1990-2010: burden of diseases, injuries, and risk factors. JAMA 310: 591-608.

2. Goldsmith SK, Pellmar TC, Kleinman AM, Bunney WE (2002) Reducing suicide: A national imperative. The National Academies Press, Washington D.C., USA.

3. Nutt DJ (2006) The role of dopamine and norepinephrine in depression and antidepressant treatment. J Clin Psychiatry 67: 3-8.

4. Palmer BA, Pankratz VS, Bostwick JM (2005) The lifetime risk of suicide in schizophrenia: a reexamination. Arch Gen Psychiatry 62: 247-253.

5. Haukka J, Suominen K, Partonen T, Lönnqvist J (2008) Determinants and outcomes of serious attempted suicide: a nationwide study in Finland, 19962003. Am J Epidemiol 167: 1155-1163.

6. Baldessarini RJ, Tondo L, Hennen J (1999) Effects of lithium treatment and its discontinuation on suicidal behavior in bipolar manic-depressive disorders. $J$ Clin Psychiatry 60: 77-84

7. Baldessarini RJ (2003) Reducing suicide risk in psychiatric disorders. Curr Psychiatry 2: 14-24.

8. Meltzer HY, Alphs L, Green Al, Altamura AC, Anand R, et al. (2003) Clozapine treatment for suicidality in schizophrenia: international suicide prevention trial (InterSePT). Arch Gen Psychiatry 60: 82-91.

9. Calabrese JR, Keck PE Jr, Macfadden W, Minkwitz M, Ketter TA, et al. (2005) A randomized, double-blind, placebo-controlled trial of quetiapine in the treatment of bipolar I or II depression. Am J Psychiatry. 162: 1351-1360.

10. Rahn KA, Cao YJ, Hendrix CW, Kaplin AI (2015) The role of 5-HT1A receptors in mediating acute negative effects of antidepressants: implications in pediatric depression. Transl Psychiatry 5: e563.

11. Zarate CA Jr, Singh JB, Carlson PJ, Brutsche NE, Ameli R, et al. (2006) A randomized trial of an $\mathrm{N}$-methyl-D-aspartate antagonist in treatment-resistant major depression. Arch Gen Psychiatry 63: 856-864.

12. Kashani P, Yousefian S, Amini A, Heidari K, Younesian S, et al. (2014) The effect of intravenous ketamine in suicidal ideation of emergency department patients. Emerg (Tehran) 2: 36-39. 